\title{
Flujo de savia y potencial hídrico en plantas de tomate (Solanum lycopersicum L.) bajo condiciones de invernadero
}

\section{Sap flow and water potential in tomato plants (Solanum lycopersicum L.) under greenhouse conditions}

CRISTIAN ALEJANDRO CUELLAR-MURCIA' JUAN CARLOS SUÁREZ-SALAZAR'1, 2

Plantas de tomate tipo chonto cultivadas bajo condiciones de invernadero en el piedemonte amazónico.

Foto: J.C. Suarez-Salazar

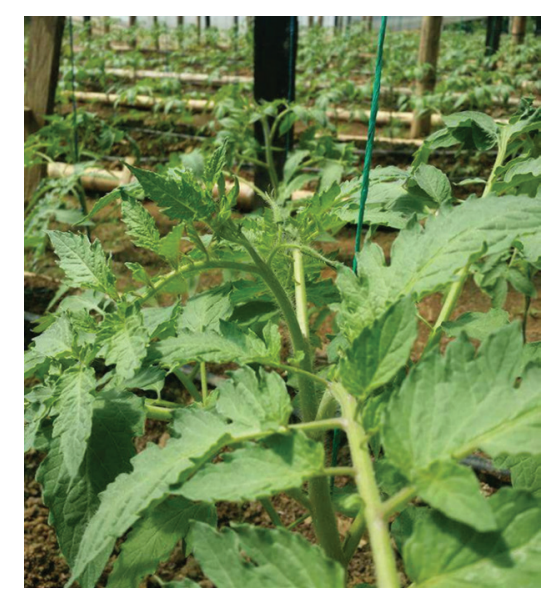

\section{RESUMEN}

El tomate es una de las hortalizas más importantes en el mundo, constituye un gran escalafón en la producción hortícola, en Colombia se reportó un área cultivada de 8.992 ha con una producción de 345.291 t. Este cultivo se desarrolla en su mayoría bajo condiciones controladas (invernaderos) requiriendo ciertos volúmenes de agua que puede ser limitantes al no realizar un monitoreo del estatus hídrico, siendo este último, información para la programación del riego. Por ello con el objeto de predecir el comportamiento el potencial hídrico del xilema $(\Psi)$ y flujo de savia $\left(F_{H 2 O}\right)$ en relación a las variables ambiéntales $\left(R A F A, H R_{\alpha} T_{\alpha} D P V\right)$ se utilizó un modelo mecánico de flujo de agua en plantas de tomate (Solanum lycopersicum L.) bajo condiciones de invernadero en el piedemonte amazónico colombiano (Florencia, Caquetá). Las tendencias diarias monitoreadas se mantuvieron entre los 64,7 a $225,4 \mathrm{~g} \mathrm{~h}^{-1} \mathrm{y}-1,2$ a -0,34 MPa para $F_{H 2 \mathrm{O}}$ Y $\Psi$ respectivamente, al modelar el comportamiento de las variables estas fueron entre rangos de -0.38 a $-1.30 \mathrm{MPa}$ para $\Psi$ y 58,46 a 208,55 g $\mathrm{h}^{-1}$ para $F_{H 2 \mathrm{O}}$, siendo estos altamente correlacionados $(P<0,0001)$. El uso del modelo mecánico de flujo de agua en plantas de tomate bajo condiciones de invernadero demostró ser estadística y fisiológicamente viable para para entender la demanda hídrica diaria el cual dependió de las variables ambientales.

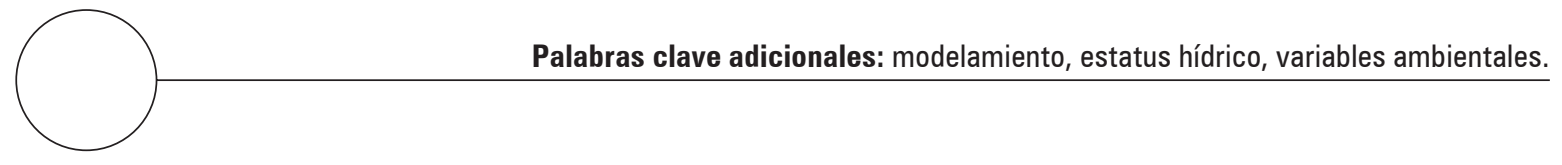

\footnotetext{
1 Facultad de Ingeniería, Universidad de la Amazonia, Florencia (Colombia). ORCID Cuellar-Murcia, C.A.: 0000-0001-8982-8629; ORCID Suárez-Salazar, J.C.: 0000-0001-5928-1837

2 Autor para correspondencia. juansuarez1@gmail.com
} 


\section{ABSTRACT}

The tomato is one of the most important horticultural fruits in the world, with large scale horticultural production in Colombia, as seen in the cultivated area of 8,992 and 345,291 t produced. The development of this crop requires production areas under controlled conditions (greenhouses) because it is important to monitor the water status of the plants to achieve successful development. In order to predict the behavior of the water potential of xylem $(\Psi)$ and sap flow $\left(\mathrm{F}_{\mathrm{H} 2 \mathrm{O}}\right)$ in relation to environmental variables $\left(R A F A, H R_{\alpha} T_{\alpha} D P V\right)$, a mechanical model of water flow in tomato plants (Solanum lycopersicum L.) was used under greenhouse conditions in Colombian Amazon piedmont (Florencia, Caquetá). The daily-monitored trends remained between 64.7 and $225.4 \mathrm{~g} \mathrm{~h}^{-1}$ and -1.2 to $-0.34 \mathrm{MPa}$ for $F_{\mathrm{H} 2 \mathrm{O}}$ and $\Psi$, respectively. To model the behavior of the variables, these trends were between -0.38 and $-1.30 \mathrm{MPa}$ for $\Psi$ and 58.46 and $208.55 \mathrm{~g} \mathrm{~h}^{-1}$ for $F_{\mathrm{HZO}}$, which were highly correlated $(P<0,0001)$. The use of a mechanical model of water flow in tomato plants under greenhouse conditions proved to be statistically and physiologically feasible for understanding the daily water demand and so can be a source of information when designing irrigation plans.

Additional key words: modeling, water status, environmental variables.

Fecha de recepción: 13-10-2017 Aprobado para publicación: 30-01-2018

INTRODUCCIÓN

El tomate es una de las hortalizas más importantes en el mundo, constituye un gran escalafón en la producción hortícola, con alrededor de 4,7 millones de hectáreas sembradas y 163,9 millones de toneladas de frutos cosechados, donde se destacan principalmente cuatro países productores; China, India, Turquía y Nigeria con una participación del 20,9; 18,8; 6,6 y 5,8\%, respectivamente (FAOSTAT, 2015). Según el DANE (2016) para el año 2015 en Colombia se reportó un área cultivada de 8.992 ha con una producción de 345.291 t. Para el desarrollo de este cultivo se requiere áreas de producción bajo condiciones controladas (invernaderos) para controlar variables ambientales como también el recurso hídrico. Este último se hace necesario monitorear para conocer el estatus hídrico ya que provee información para la programación del riego (Meng et al., 2017).

El estatus hídrico de la planta de tomate es monitoreado mediante el flujo de savia y el potencial hídrico (De Swaef et al., 2012) que expresan la velocidad y fuerza del agua, los cuales dependen del proceso de transpiración (Ballester et al., 2013), afectando el balance hídrico. Este proceso fisiológico es fluctuante durante el día debido a los cambios en la radiación, la precipitación, la temperatura, la humedad del aire y entre otros factores (Both et al., 2015). Monitorear los cambios de estatus hídrico es una herramienta de suma importancia a la hora de establecer programas de riego los cuales pueden ser obtenidos mediante modelos matemáticos utilizando como variables de respuesta el flujo de savia y el potencial hídrico junto con las variables ambientales (De Swaef y Steppe, 2010; De Swaef et al., 2013).

Una adecuada explicación del comportamiento del flujo de agua en especies vegetales mediante modelos matemáticos ha sido de amplio interés (De Swaef et al., 2010, 2012, 2014; Steppe et al., 2005, 2010), implicando una exhaustiva validación de los modelos por medio de pruebas experimentales (De Swaef et al., 2015). En algunos estudios de especies leñosas se ha explicado exitosamente el estatus hídrico por medio de predicciones hechas a partir de las mediciones diarias del flujo de savia, el diámetro de tallo y el potencial hídrico del tallo y suelo (Steppe et al., 2005; Verbeeck et al., 2007; Vandegehuchte et al., 2014), siendo igualmente explicado en el cultivo del tomate (De Swaef y Steppe, 2010; De Swaef et al., 2013). En este sentido, el uso de modelos de predicción es capaz de ajustarse a diferentes especies y condiciones ambientales para expresar el estado hídrico en las plantas (De Swaef et al., 2014). Por lo anterior, el objetivo del presente estudio fue predecir el comportamiento del flujo de savia y potencial hídrico en relación a las variables ambiéntales en plantas de tomate bajo 
condiciones de invernadero en el piedemonte amazónico colombiano (Florencia, Caquetá) mediante un modelo mecánico de flujo de agua.

\section{MATERIALES Y MÉTODOS}

\section{Localización del área de estudio}

Las mediciones se realizaron en plantas de tomate en el Centro de Investigaciones CIMAZ Macagual, Universidad de la Amazonía, Colombia (1³7' N y 75³6' W). El clima es de tipo cálido-húmedo, característico del ecosistema de bosque húmedo tropical con una altitud de $326 \mathrm{msnm}$, una precipitación anual promedio de $3.800 \mathrm{~mm}$, un brillo solar de $1.700 \mathrm{~h}$ año ${ }^{-1}$, una temperatura promedio de $25,5^{\circ} \mathrm{C}$ y humedad relativa del aire del $84 \%$. Las plantas de tomate fueron sembradas en un invernadero con una densidad de 12 plantas $/ \mathrm{m}^{2}$, manejado por un sistema de riego por goteo y microaspersión, con aplicaciones diarias durante todo el monitoreo de 4 y $4,5 \mathrm{~mm}$ respectivamente. El material de tomate fue tipo chonto (Solanum lycopersicum L.), híbrido certificado denominado súper Daniela producido por el laboratorio Hanzera Genetics, cuyo ciclo promedio de vida es de 130 a 180 $\mathrm{d}$, una madurez relativa tardía, un vigor fuerte y una adaptabilidad desde los 300 a los $2.600 \mathrm{msnm}$. Para la etapa de establecimiento del cultivo, se usaron dos fuentes de fertilización, una de tipo orgánico (gallinaza) y otra de síntesis química (15-15-15), con una dosificación de 0,3 y $0,1 \mathrm{~kg} /$ planta respectivamente, posteriormente se redujo al $30 \%$ con periodos mensuales de aplicación.

\section{Monitoreo de variables ambiéntales, potencial hídrico y flujo de savia}

El monitoreo de las variables ambiéntales y fisiológicas se realizó en el periodo comprendido entre los días julianos 94 (abril 4) al 101 (abril 11) del año 2015, época fenológica correspondiente a la de llenado de fruto. Para el monitoreo de las variables ambientales al interior del invernadero, se utilizó una estación meteorológica marca WatchDog 2900ET (Spectrum Technologies, Aurora, IL, USA) con una frecuencia de minuto a minuto para determinar la humedad relativa $\left(H R_{a}, \%\right)$, la temperatura del aire $\left(T_{a},{ }^{\circ} \mathrm{C}\right)$, y la radiación fotosintéticamente activa (RAFA, $\mu \mathrm{mol}$ fotones $\left.\mathrm{m}^{-2} \mathrm{~s}^{-1}\right)$. Se calculó el déficit presión de vapor $(D P V$,
$\mathrm{kPa}$ a partir de los valores máximos y mínimos de la temperatura del aire y de la humedad relativa registrada hora a hora, siguiendo la metodología propuesta por Allen et al. (2006).

El potencial hídrico del xilema ( $\Psi, \mathrm{MPa}$ ) se determinó en plantas de tomate bajo condiciones de invernadero utilizando un sensor tipo PSY1 (Stem Psychrometer ICT International, Armidale, NSW, Australia) en el tallo principal, a una altura de $20 \mathrm{~cm}$. Para hacer la inserción del sensor fue necesario retirar cuidadosamente las capas de tejido de la corteza utilizando una cuchilla para exponer la capa del tejido xilematico. El sensor se fijó al xilema conductor expuesto y se utilizó grasa de silicona para sellar alrededor del psicrómetro y cubrir cualquier tejido expuesto (Patankar et al., 2012).

Para la medición del flujo de savia $\left(F_{\mathrm{H} 2 \mathrm{O}} ; \mathrm{g} \mathrm{h}^{-1}\right)$ se instaló un sensor tipo SFM1 (Sap Flow Meter ICT International, Armidale, NSW, Australia) en el tallo principal de la planta de tomate, a una altura de $20 \mathrm{~cm}$ del suelo, los cuales se basan en el principio del método pulso de calor (Heat Ratio Method, HRM). Este método ha sido ampliamente utilizados como herramienta para medir el uso del agua por las plantas (Steppe et al., 2010; Miner et al., 2017), permitiendo cuantificar la velocidad del flujo de savia en base a curvas de temperatura a partir de un impulso de calor (densidad y difusión térmica). El sensor SFM1 está compuesto por dos agujas y una sonda de calor integrada a un microprocesador para determinar $V_{s}$. Cada aguja está compuesta por dos termocuplas las cuales reciben el pulso de calor que incrementa la temperatura en el xilema conductor y que es proporcional a la velocidad del pulso de calor $\left(V_{s}, \mathrm{~g} \mathrm{~h}^{-1}\right)$ (Burgess et al., 2001).

La $V_{s}$, se calculó según la siguiente equación:

$$
V_{s}=\frac{k}{x} \ln \left(V_{1} / V_{2}\right) \times 3600
$$

Dónde: $k$ es la difusividad térmica en el área del xilema conductor (se asumió un default value of $0,0025 \mathrm{~cm}^{2}$ $\left.\mathrm{s}^{-1}\right), x$ es la distancia entre la sonda de calor y cada una de las agujas $(\mathrm{cm}), V$ es la aumento de la temperatura $\left({ }^{\circ} \mathrm{C}\right)$ inicial con respecto a la aguja ubicada en la parte inferior $\left(V_{1}\right)$ y superior $\left(V_{2}\right)$.

Los sensores SMF1 y PSY1 estuvieron acoplados a un sistema de recolección de datos independiente (CR10X datalogger + multiplexor AM416, Campbell 
Scientific, Logan, UT, USA) el cual almaceno una medida cada quince minutos, desde las 0:00 hasta las 23:00 h.

\section{Modelo predictivo y análisis de datos}

El modelo utilizado para predecir el flujo de agua fue el propuesto Steppe et al. (2005), usado principalmente para plantas leñosas y posteriormente adaptado de De Swaef y Steppe (2010) para plantas herbáceas como el tomate (De Swaef et al., 2013). En el modelo se incluyeron los parámetros expuestos en la tabla 1, utilizados para la simular el potencial hídrico del xilema ( $\Psi$ ) y el flujo de agua en el tallo $\left(F_{H_{2} \mathrm{O}}\right)$ durante ocho días consecutivos.

Posteriormente de haber concretado los parámetros, se dio inicio a usar las ecuaciones adaptadas de De Swaef y Steppe (2010) (Tab. 2), las cuales relaciona la dinámica del flujo de agua en plantas herbáceas, bajo el concepto de resistencia al flujo, a partir de una variable de entrada que es el flujo se savia $\left(F_{H 2 O}\right)$ evaluada durante el respetivo monitoreo. Para el análisis de los datos se usó el software estadístico InfoStat (Di Rienzo et al., 2017), donde se aplicó el análisis de estadística descriptiva para cada variable y análisis de correlación de Pearson entre las variables fisiológicas ( $\Psi$ y $F_{\mathrm{H} 2 \mathrm{O}}$ ) y variables ambientales (RAFA, $D P V, T_{a}$ y $\left.H R_{a}\right)$ y entre las variables fisiológicas monitoreadas y simuladas, como regresiones para determinar los respectivos comportamientos durante el monitoreo.

\section{RESULTADOS Y DISCUSIÓN}

\section{Comportamiento de variables ambientales}

La variables ambientales obtuvieron rangos diarios entre los 0 a $1.248,5 \mu \mathrm{mol}$ fotones $\mathrm{m}^{-2} \mathrm{~s}^{-1}$ para $R A F A$, 53,48 a $99,9 \%$ para $H R_{a}, 22,1$ y $33,15^{\circ} \mathrm{C}$ para $T_{a}$ y de 0,001 y $1,81 \mathrm{kPa}$ para $D P V$ siendo mayor a las $13: 00 \mathrm{~h}$ para $R A F A, D P V y T_{a}$, sin embargo, $H R_{a}$ se presentó lo contrario. Al analizar el comportamiento de las variables ambientales durante el periodo de monitoreo se encontró periodos máximos promedios, como el presentado en el primer día del estudio (día 94), reportando valores promedio de $259,8 \pm 77,5 \mu \mathrm{mol}$ fotones $\mathrm{m}^{-2}$ $\mathrm{s}^{-1}$ para $R A F A, 25,9 \pm 0,8^{\circ} \mathrm{C}$ para $T_{a}, 0,5 \pm 0,1 \mathrm{kPa}$ para $T_{a}$ y $85,36 \pm 3,70 \%$ para $H R_{a}$. Contrario a lo presentado al octavo día del estudio (día 101) donde por efectos de precipitación se redujo en mayor medida las horas luz, incidiendo sobre las variables ambientales, llegando a valores promedios de $68,18 \pm 23,39 \mu \mathrm{mol}$ fotones $\mathrm{m}^{-2}$ $\mathrm{s}^{-1}$ para $R A F A, 23,09 \pm 0,25^{\circ} \mathrm{C}$ para $T_{a}, 0,10 \pm 0,03 \mathrm{kPa}$ para $T_{a}$ y $96,09 \pm 1,17 \%$ para $H R_{a}$ (Fig. 1 ), siendo los menores valores durante todo el periodo del estudio.

Tabla 1. Parámetros empleados en el modelo mecánico de flujo de agua en plantas herbáceas adaptado por De Swaef y Steppe (2010).

\begin{tabular}{|c|c|l|c|l|}
\hline Parámetro & Unidad & \multicolumn{1}{|c|}{ Definición } & \multicolumn{1}{|c|}{ Valor } & \multicolumn{1}{c|}{ Origen } \\
\hline$R^{\times}$ & $\mathrm{MPa} \mathrm{h} \mathrm{g}^{-1}$ & Resistencia hidráulica entre el suelo y el tallo & 0,00573 & De Swaef y Steppe (2010) \\
\hline$\Psi_{\text {(sustrato) }}$ & $\mathrm{MPa}$ & Potencial hídrico del sustrato & $-0,005$ & De Swaef y Steppe (2010) \\
\hline & $\mathrm{g} \mathrm{h}^{-1}$ & Flujo de savia en el tallo & - & Este estudio \\
\hline & $\mathrm{MPa}$ & Potencial hídrico del xilema conductor & - & Este estudio \\
\hline
\end{tabular}

Tabla 2. Modelo mecánico de flujo de agua adaptado de De Swaef y Steppe (2010) para plantas herbáceas, donde: $\Psi_{(\text {(sustrato) }}$ : potencial hídrico del sustrato, $R^{x}$ : resistencia hidráulica entre el suelo y el tallo, $F_{\mathrm{H}_{2}}$ : flujo de agua en el tallo, $\Psi$ : potencial hídrico del xilema.

\begin{tabular}{|l|c|c|}
\hline \multicolumn{1}{|c|}{ Descripción } & Ecuación & Unidad resultado \\
\hline Flujo de savia en el tallo & $\mathrm{F}_{\mathrm{H}_{2} \mathrm{O}}=-\frac{\Psi-\Psi_{\text {(sustrato) }}}{R^{x}}$ & $\mathrm{~g} \mathrm{~h}{ }^{-1}$ \\
\hline Potencial hídrico del xilema conductor & $\Psi=\Psi_{\text {(sustrato) }}-\mathrm{F}_{\mathrm{H}_{2} \mathrm{O}^{*}} R^{\times}$ & $\mathrm{MPa}$ \\
\hline
\end{tabular}




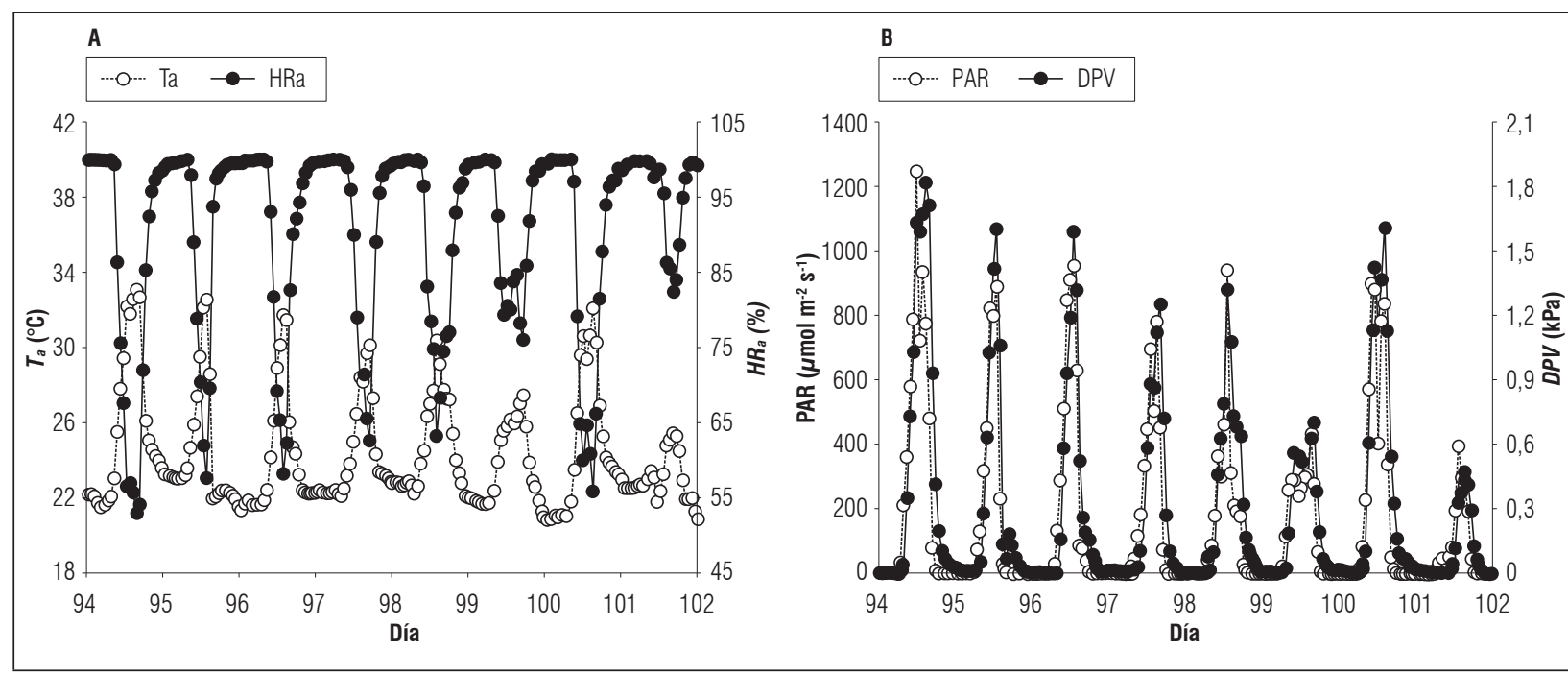

Figura 1. Comportamiento diario de las variables ambiéntales. A. Temperatura del aire $\left(T_{a}\right)$ y Humedad relativa $\left(H R_{a}\right)$; $B$. Radiación fotosintéticamente activa (PAR) y déficit presión de vapor (DPV); durante los días 94 y 101 del 2015 bajo condiciones de invernadero.

\section{Dinámica del potencial hídrico $(\Psi)$}

La tendencia diaria del $\Psi$ no expreso valores limitantes (<-1,5 MPa, Zegbe et al., 2006) en el estatus hídrico, ya que se mantuvo entre los $-0,34$ a $-1,2 \mathrm{MPa}$, presentando durante el monitoreo valores más negativos al medio día (Fig. 2A). La tendencia de $\Psi$ presentado en el cultivo de tomate fue similar a la reportada por Zhang et al. (2017) quienes mencionan valores entre $-0,23$ y $-1,03 \mathrm{MPa}$ cuando valores de DPV estuvo alrededor de 1 a $2 \mathrm{kPa}$. Al ser el $\Psi$ de la planta menos negativo se reduce sustancialmente la fuerza motriz para el transporte de agua y por lo tanto se disminuye su perdida y a su vez se regula el estrés hídrico (Fricke, 2016). Tales variaciones diarias del $\Psi$ en la planta son ocasionada principalmente a los déficits hídricos presentes en la atmosfera y en el suelo, siendo consecuencia de las condiciones microclimáticas (Martínez et al., 2014). El agua en el suelo es una de las variables que más fuerte se relaciona con el $\Psi$, provocando una fluctuación directa durante el día (Vandegehuchte et al., 2014). Aunque en este estudio no se evaluó el contenido de agua en el suelo, en algunas investigaciones se ha observado el efecto positivo sobre una adecuada humedad en el mismo, el cual da como resultado un óptimo $\Psi$, y por consiguiente la inexistencia del déficit hídrico o viceversa (Ismail, 2010; Quintal et al., 2012). De esta forma el agua presente en el suelo expresa un efecto positivo sobre el crecimiento celular y sobre la elasticidad de las paredes celulares de las plantas, atributos que ayudan al aprovechamiento del agua y a optimizar procesos dependientes como el flujo de savia y la fotosíntesis (Silva et al., 2012; Both et al., 2015), por tal razón lo valores se mantuvieron en rangos no limitantes.

\section{Dinámica del flujo de savia $\left(\mathrm{F}_{H 20}\right)$}

La tendencia diaria del $F_{H 2 O}$ durante el monitoreo fue entre 64,7 a 22,4 $\mathrm{g} \mathrm{h}^{-1}$, aumentando desde las 6:00 hasta las 13:00 horas, sin embargo, en las horas de la tarde se reduce el flujo de savia (Fig. 2B). El comportamiento diario de $F_{\mathrm{H} 2 \mathrm{O}}$ fue similar al reportado por Guangcheng et al. (2016) quienes en una evaluación a $80 \%$ capacidad de campo, el flujo fue savia incremento en la mañana hasta llegar a su punto máximo promedio de 297,3 $\mathrm{g} \mathrm{h}^{-1}$ en la hora 13:00, siendo este el periodo de mayor demanda hídrica. Posterior a esta hora los valores disminuyen debido al cierre estomático existiendo una reducción en la actividad transpiratoria, manteniendo así un equilibrio entre la tasa de captación de agua por la raíz y la tasa de transpiración (Gong et al., 2001; Liu et al., 2007; Qiu et al., 2015).

Durante la noche, en todo el periodo de monitoreo el flujo fue positivo, movilizando el 30,9\% del agua transportada por la planta durante todo el día, con valores entre los 64,7 a 76,6 $\mathrm{g} \mathrm{h}^{-1}$. Los valores nocturnos en esta especie expresaron un comportamiento activo durante dicho periodo, debido a que sus estomas experimentaron un cierre parcial o incompleto, 

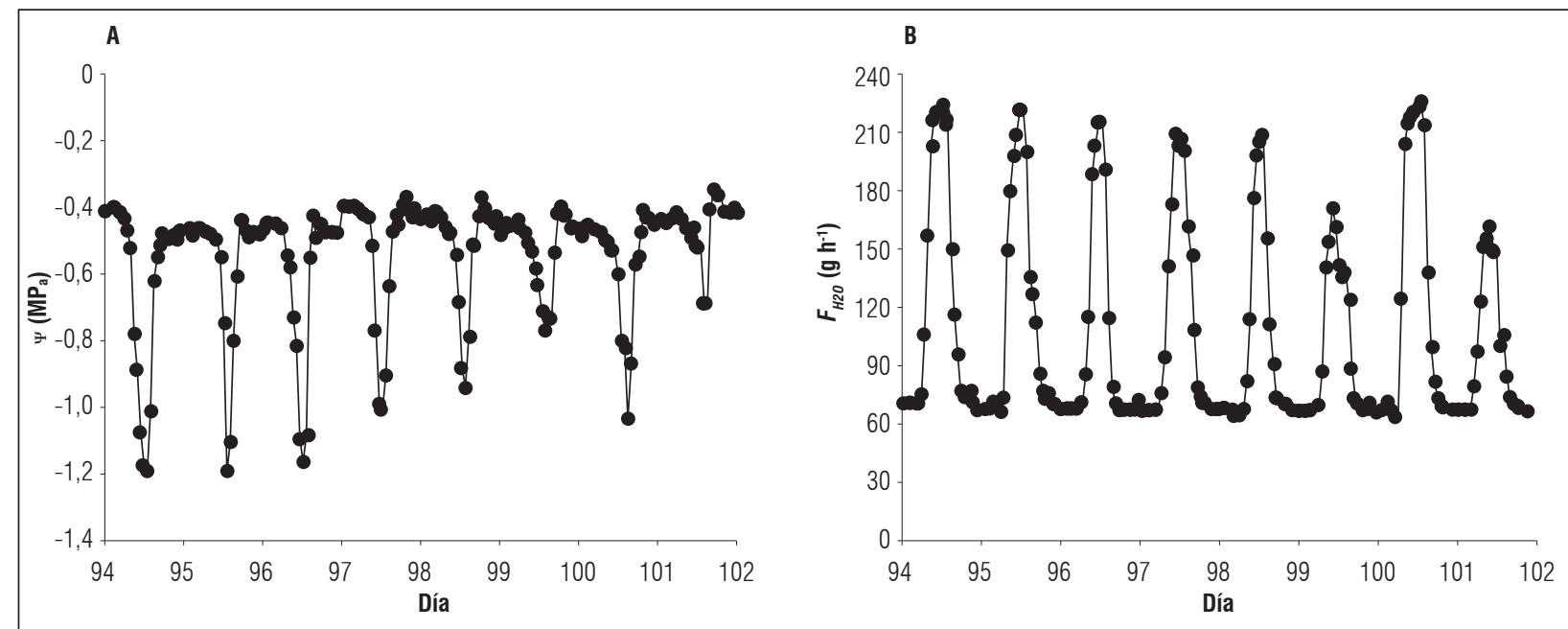

Figura 2. Comportamiento diario del potencial hídrico del xilema conductor $(\Psi, \mathrm{A})$ y el flujo de savia $\left(\boldsymbol{F}_{H 20}\right.$, B) en plantas de tomate durante los días 94 y 101 del 2015 bajo condiciones de invernadero.

manteniendo así valores del flujo de agua positivos (Easlon y Richards, 2009). Este comportamiento se ha podido encontrar en muchas especies, donde los principales responsables son el transporte y pérdida de agua por consecuencia de la transpiración nocturna (Caird et al., 2007; Qiu et al., 2015). Las cantidades diarias reportadas se pueden atribuir a los volúmenes de agua presente en el suelo, donde el principal responsable de dichos volúmenes fueron los eventos de riego durante los días de evaluación, siendo una condición importante para mejorar los niveles de flujo de agua en esta especie (Guangcheng et al., 2016).

\section{Relación entre las variables ambientales y fisiológicas}

Las tendencias obtenidas en este trabajo expresan como el $\Psi$ y el $F_{H 2 O}$ se relacionaron con las variables ambientales durante el periodo de monitoreo. El $\Psi$ obtuvo la menor explicación estadística frente al $F_{H 2 O}$, presentando así correlación negativa con RAFA $(-0,78 ; P<0,0001), T_{a}(-0,69 ; P<0,0001)$ y $D P V(-0,72$; $P<0,0001)$ y positiva para $H R_{a}(0,71 ; P<0,0001)$. Por el contrario, el $F_{H 2 O}$ presento correlación positiva con RAFA (0,87; $\mathrm{P}<0,0001), T_{a}(0,82 ; \mathrm{P}<0,0001)$ y $D P V(0,81 ; P<0,0001)$ y negativa para $H R_{a}(-0,83$; $P<0,0001)$. La poca correlación de $\Psi$ es explicada por las fluctuaciones de las variables ambientales en el transcurso del monitoreo afectado principalmente por la radiación $(R A F A)$ y del déficit presión de vapor (DPV, García et al., 2010; Zhang et al., 2017).
A su vez, el $\Psi$ es afectado por otros factores como el contenido de agua en el suelo y en la atmosfera (Martínez et al., 2014; Fricke, 2016).

Por otro lado, el $F_{H 2 O}$ al ser la variable con mayor explicación estadística y correlación frente a las variables ambientales, expreso mayor relación con RAFA y $D P V$ (Qiu et al., 2015). El DPV es una de las variables ambientales de mayor importancia a la hora de establecer cultivos en invernaderos, debido a que sus variaciones regulan la temperatura y la humedad relativa ambiental, siendo dos factores esenciales para mantener niveles adecuados de agua en el suelo y en la atmósfera (Zhu et al., 2010; Both et al., 2015). De esta forma repercute directamente en la absorción y transporte de líquidos a través de la planta, procesos esenciales para el incremento de la actividad fotosintética y de la productividad (Guangcheng et al., 2016). Un DPV elevado (mayor a -1,5 MPa) implica una respuesta fisiológica inmediata en la planta, donde sus estomas expresarían un cierre parcial para evitar las pérdidas de agua, principalmente en forma de vapor (Qiu et al., 2015; Zhang et al., 2017). En consecuencia, se afectarían procesos esenciales como el intercambio gaseoso, la actividad fotosintética y el rendimiento de la planta, siendo este último de gran importancia económica, dado que se reduce el potencial productivo de la especie (Bobich et al., 2010). Caso contrario es visto para un $D P V$ bajo y optimo en el cual la planta aumentaría el desempeño de sus actividades fisiológicas (Zhang et al., 2017). 


\section{Predicciones}

En la figura 3 se observan la modelación de $\Psi$ y $F_{H 2 O}$ durante el periodo de monitoreo cuyos rangos fueron entre $-0,38$ y $-1,30$ MPa para $\Psi$ y con 58,46 y 208,55 $\mathrm{g} \mathrm{h}^{-1}$ para $F_{\mathrm{H} 2 \mathrm{O}}$. Los resultados obtenidos de las modelaciones se encuentran entre los rangos reportados por la literatura, compartiendo las mismas tendencias diarias obtenidos en otros estudios (De Swaef y Steppe, 2010; De Swaef et al., 2013; Qiu et al., 2015; Guangcheng et al., 2016; Zhang et al., 2017), a su vez se encontró una alta correlación $(0,82 ; P<0,0001)$.

La amplia correlación se debe a la dependencia entre sí de dichas variables para expresar el estado hídrico en la planta. A la medida que el $F_{H 2 O}$ aumenta el $\Psi$ se vuelve más negativo y viceversa, expresando de esta forma una mayor fuerza motriz para el transporte de agua a través del tallo (Steppe et al., 2005), siendo consecuencia directa de tres factores; la transpiración, el volumen de agua presente en el suelo y la demanda atmosférica (Vandegehuchte et al., 2014; Martínez et al., 2014; Oiu et al., 2015). Los eventos de riego además de regular las condiciones ambiéntales aumentaron la disponibilidad de agua, siendo resultado del incremento directo del gradiente de potencial de agua entre el suelo y los tejidos de almacenamiento del ta1lo, por lo cual dichos tejidos se reponían y aumentaban así el nivel de agua transportada por la planta (De Swaef y Steppe, 2010). Tal tendencia ha sido explicada por medio del concepto de resistencia al flujo, donde se expresa como el gradiente del potencial hídrico varia directamente por el contenido de agua presente en los tejidos de almacenamiento, generando así una mayor tensión en el xilema que impulsara el flujo vertical de agua a través del tallo (De Swaef et al., 2013). Tal como se ha visto en especies leñosas (Steppe et al., 2005) y en herbáceas (De Swaef y Steppe, 2010) con los ajustes y resultados exitosos respectivos. De igual forma en este estudio el modelo se aplicó con resultados satisfactorios para entender el estado hídrico diario de esta especie en relación a la respuesta fisiológica derivada de la demanda atmosférica.

\section{CONCLUSIONES}

El modelo mecánico utilizado en plantas de tomate bajo condiciones de invernadero tuvo un alto nivel de predicción $(r=0,82 ; P<0,0001)$ del potencial hídrico del xilema $(\Psi)$ y flujo de savia $\left(F_{H 2 O}\right)$. Así mismo se encontró que el comportamiento diario de $F_{H 2 O}$ fue sensiblemente afectado de manera positiva por las variables ambientales basado en las correlaciones obtenidas con $R A F A(0,87 ; P<0,0001), T_{a}(0,82 ; P<0,0001)$ y $D P V(0,81 ; P<0,0001)$ y negativa para $H R_{a}(-0,83$; $P<0,0001)$, aumentando desde las 6:00 hasta las 13:00 h, sin embargo en las horas de la tarde se reduce el flujo de savia. Así mismo, el $\Psi$ fue afectado de manera negativa por RAFA $(-0,78 ; P<0,0001), T_{a}(-0,69$; $P<0,0001)$ y $D P V(-0,72 ; P<0,0001)$ y positiva para $H R_{a}(0,71 ; P<0,0001)$.
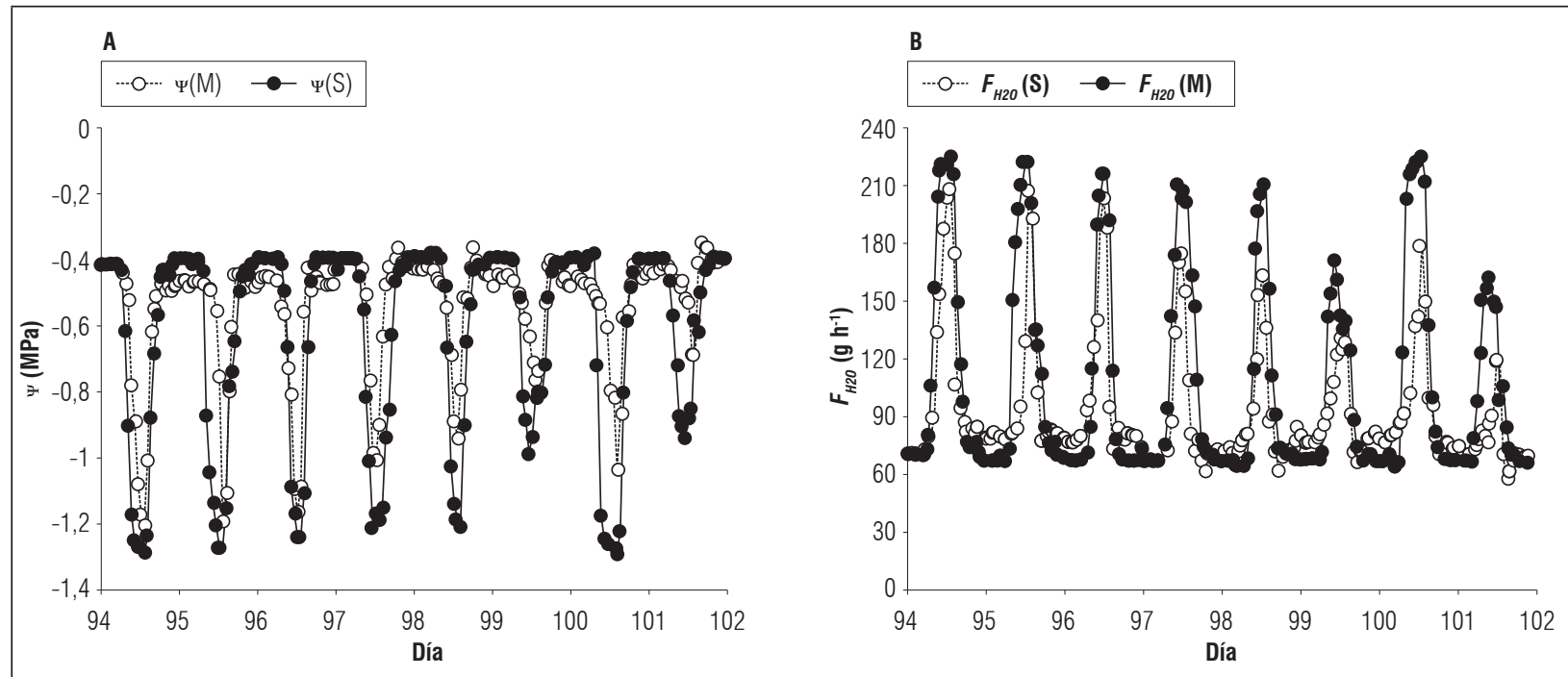

Figura 3. Comportamiento del potencial hídrico del xilema $(\Psi, A)$ y flujo de savia $\left(F_{\mathrm{H} 20}, B\right)$ en plantas de tomate bajo condiciones de invernadero. M: monitoreada, S: simulada. 
Conflicto de intereses: el manuscrito fue preparado y revisado con la participación de los autores, quienes declaran no tener algún conflicto de interés que coloquen en riesgo la validez de los resultados aquí presentados.

\section{REFERENCIAS BIBLIOGRÁFICAS}

Allen, R., S. Pereira, D. Raes y M. Smith. 2006. Evapotranspiración del cultivo: Guías para determinación los requerimientos de agua de los cultivos. Estudio Riego e Drenaje 56. FAO, Roma, Italia

Ballester, C., J. Castel, L. Testi, D. Intrigliolo y J.R. Castel. 2013. Can heatpulse sap flow measurements be used as continuous water stress indicators of citrus trees?. J. Irrig Sci. 31, 1053-1063. Doi: 10.1007/ s00271-012-0386-5

Bobich, E., G. Barron, K. Rascher y R. Murthy. 2010. Effects of drought and changes in vapour pressure deficit on water relations of Populus deltoides growing in ambient and elevated CO. Tree Physiol. 30, 866-875. Doi: 10.1093/treephys/tpq036

Both, A., L. Benjamin, J. Franklin, G. Holroyd, L. Incoll, M. Lefsrud y G. Pitkin. 2015. Guidelines for measuring and reporting environmental parameters for experiments in greenhouses. Plant Methods 11, 43-68. Doi: 10.1186/s13007-015-0083-5

Burgess, S., M. Adams, N. Turner. C, Beverly. C. Ong, A. Khan y T. Bleby. 2001. An improved heat pulse method to measure low and reverse rates of sap flow in woody plants. Tree physiology. 21(9): 589-598. Doi: 10.1093/treephys/21.9.589

Caird, M., J. Richards y T. Hsiao. 2007. Significant transpirational water loss occurs throughout the night in field-grown tomato. Funct. Plant Biol. 34, 172-177. Doi: 10.1071/FP06264

DANE (Departamento Administrativo Nacional de Estadística) 2016. Encuesta nacional agropecuaria ENA 2015. Cód.: DIE-020-PD-01-r5_v7. Bogotá, Colombia

De Swaef, T., V. De Schepper, M. Vandegehuchte y K. Steppe. 2015. Stem diameter variations as a versatile research tool in ecophysiology. Tree Physiol. 35(10), 1047-1061. Doi: 10.1093/treephys/tpv080

De Swaef, T., J. Hanssens, A. Cornelis y K. Steppe. 2013. Non-destructive estimation of root pressure using sap flow, stem diameter measurements and mechanistic modelling. Ann. Bot. 111, 271-282. Doi: 10.1093/aob/ mcs249

De Swaef, T., C. Mellisho, A. Baert, V. De Schepper. A. Torrecillas, W. Conejero y K. Steppe. 2014. Model-assisted evaluation of crop load effects on stem diameter variations and fruit growth in peach. Trees 28(6), 1607-1622. Doi: 10.1007/s00468-014-1069-z
De Swaef, T. y K. Steppe. 2010. Linking stem diameter variations to sap flow, turgor and water potential in tomato. Funct. Plant Biol. 37, 429-438. Doi: 10.1071/ FP09233

De Swaef, T., K. Verbist, W. Cornelis y K. Steppe. 2012. Tomato sap flow, stem and fruit growth in relation to water availability in rockwool growing medium. Plant Soil 350(1-2), 237-252. Doi: 10.1007/ s11104-011-0898-4

Di Rienzo, J., F. Casanoves, M. Balzarini, L. Gonzalez, M. Tablada y C. Robledo. 2017. Infostat versión 2017. Grupo Infostat, FCA, Universidad Nacional de Córdoba, Córdoba, Argentina.

Easlon, H. y J. Richards. 2009. Drought response in self-compatible species of tomato (Solanaceae). Amer. J. Bot. 96, 605-611. Doi: 10.3732/ajb.0800189

FAOSTAT. 2015. Production/yield quantities of tomatoes in world. En: FAOSTAT. http://www.fao.org/faostat/ en/\#data/OC; consulta: agosto de 2017.

Fricke, W. 2016. Water transport and energy. Plant Cell Environ. 40, 977-994. Doi: 10.1111/pce.12848

García, A., R. Cun y L. Montero. 2010. Efecto de la hora del día en el potencial hídrico foliar del sorgo y su relación con la humedad en el suelo. Rev. Cienc. Téc. Agropecu. 19(3), 7-11.

Gong, D., J. Wang y S. Kang. 2001. Variations of stem and root sap flow of peach tree under different water status. Transactions of the CSAE 17(4), 33-37.

Guangcheng, S., H. Doudou, C. Xi, C. Jingtao y Z. Zhenhua. 2016. Path analysis of sap flow of tomato under rain shelters in response to drought stress. Int. J. Agric. Biol. Eng. 9(2), 54-62.

Ismail, S. 2010. Influence of deficit irrigation on water use efficiency and bird pepper production (Capsicum annum L.). Meteor. Environ. Arid Land Agric. Sci. 21, 2943. Doi: 10.4197/met.21-2.3

Liu, H., M. Genard, S. Guichard y N. Bertin. 2007. Model-assisted analysis of tomato fruit growth in relation to carbon and water fluxes. J. Exp. Bot. 58, 3567-3580. Doi: 10.1093/jxb/erm202

Martínez, J., R. Poyatos, D. Aguadé, J. Retana y M. Mencuccini. 2014. A new look at water transport regulation in plants. New Phytologist 204(1), 105-115. Doi: 10.1111/nph.12912

Meng, Z., A. Duan, D. Chen, B. Dassanayake, X. Wang, Z. Liu y S. Gao. 2017. Suitable indicators using stem diameter variation-derived indices to monitor the water status of greenhouse tomato plants. PloS one 12(2), e0171423. Doi: 10.1371/journal.pone. 0171423

Miner, G., J. Ham y G. Kluitenberg. 2017. A heat-pulse method for measuring sap flow in corn and sunflower using 3D-printed sensor bodies and 
low-cost electronics. Agric. For. Meteor. 246, 86-97. Doi: 10.1016/j.agrformet.2017.06.012

Patankar, R., W. Quinton y J. Baltzer. 2013. Permafrost-driven differences in habitat quality determine plant response to gall-inducing mite herbivory. J. Ecol. 101, 1042-1052. Doi: 10.1111/1365-2745.12101

Qiu, R., T. Du, K. Shaozhong, R. Chen y L. Wu. 2015. Influence of water and nitrogen stress on stem sap flow of tomato grown in a solar greenhouse. J. Amer. Soc. Hort. Sci. 140(2), 111-119.

Quintal, W., A. Pérez, L. Latournerie, C. May, E. Ruiz y A. Martínez. 2012. Uso de agua, potencial hídrico y rendimiento de chile habanero (Capsicum chinense Jacq.). Rev. Fitotec. Mex. 35(2), 155-160.

Silva, C., G. Sellés, R. Ferreyra y H. Silva, 2012. Variation of water potential and trunk diameter answer as sensitivity to the water availability in table grapes. Chil. J. Agric. Res. 72(4), 459-469. Doi: 10.4067/ S0718-58392012000400001

Steppe, K., D. De Pauw, T. Doody y R. Teskey. 2010. A comparison of sap flux density using thermal dissipation, heat pulse velocity and heat field deformation methods. Agric. For. Meteor. 150(7), 1046-1056. Doi: 10.1016/j.agrformet.2010.04.004

Steppe, K., D. De Pauw, R. Lemeur y P. Vanrolleghem. 2005. A mathematical model linking tree sap flow dynamics to daily stem diameter fluctuations and radial stem growth. Tree Physiol. 26, 257-273. Doi: 10.1093/ treephys/26.3.257

Vandegehuchte, M., A. Guyot, M. Hubeau, T. De Swaef, D. Lockington y K. Steppe. 2014. Modelling reveals endogenous osmotic adaptation of storage tissuewater potential as an important driver determining different stem diameter variation patterns in the mangrove species Avicennia marina and Rhizophora stylosa. Ann. Bot. 114, 667-676. Doi: 10.1093/aob/mct311

Verbeeck, H., K. Steppe, N. Nadezhdina, M. De Beeck, G. Deckmyn, L. Meiresonne y I. Janssens. 2007. Model analysis of the effects of atmospheric drivers on storage water use in Scots pine. Biogeosci. 4(4), 657-671. 10.5194/bg-4-657-2007

Zegbe, J., M. Behboudian y B. Clothier. 2006. Responses of 'Petopride'processing tomato to partial rootzone drying at different phenological stages. Irrig. Sci. 24(3), 203-210. Doi: 10.1007/s00271-005-0018-4

Zhang, D., Q. Du, Z. Zhang, X. Jiao, X. Song y J. Li. 2017. Vapour pressure deficit control in relation to water transport and water productivity in greenhouse tomato production during summer. Scient. Rep. 7, srep43461.

Zhu, X., S. Long y D. Ort. 2010. Improving photosynthetic efficiency for greater yield. Annu. Rev. Plant Biol. 61, 235-261.Doi:10.1146/annurev-arplant-042809-112206 